Full length research article

\title{
Visualizing compaction of polysomes in bacteria
}

Nicolas Cougot ${ }^{1}$, Anne-Elisabeth Molza ${ }^{1}$, Jeremy Delesques ${ }^{1}$, Emmanuel Giudice ${ }^{1}$, Annie Cavalier ${ }^{1}$, Jean-Paul Rolland ${ }^{1}$, Gwennola Ermel ${ }^{2}$, Carlos Blanco', Daniel Thomas ${ }^{1}$ and Reynald Gillet $^{1,3 *}$

${ }^{1}$ Team Translation and Folding, Université de Rennes 1, UMR CNRS 6290 IGDR, Campus de Beaulieu 35042 Rennes cedex, France

${ }^{2}$ Université de Rennes 1, EA 1254, Campus de Beaulieu 35042 Rennes cedex, France

${ }^{3}$ Institut Universitaire de France

* R. Gillet can be contacted at Team Translation and Folding, Université de Rennes 1, UMR CNRS 6290 IGDR, Campus de Beaulieu 35042 Rennes Cedex, France. E-mail address: reynald.gillet@univ-rennes1.fr

\begin{abstract}
During protein synthesis, many translating ribosomes are bound together with an mRNA molecule to form polysomes (or polyribosomes). While the spatial organization of bacterial polysomes has been well-studied in vitro, little is known about how they cluster when cellular conditions are highly constrained. To better understand this, we used electron tomography, template matching, and 3D modeling to analyze the supramolecular network of ribosomes after induction of translational pauses. In Escherichia coli, we overexpressed an mRNA carrying a polyproline motif known to induce pausing during translation. When working with a strain lacking tmRNA, the principle actor in the "trans-translation" rescuing system, the cells survived the hijacking of the translation machinery but this resulted in a sharp modification of the ribosomal network. The results of our experiments demonstrate that single ribosomes are replaced with large amounts of compacted polysomes. These polysomes are highly organized, principally forming hairpins and dimers of hairpins that stack together. We propose that these spatial arrangements help to maintain translation efficiency when the rescue systems are absent or overwhelmed.
\end{abstract}

Key words: electron tomography, mRNA, polysomes, ribosome, tmRNA

Running title: Polysomal compaction in bacteria 


\section{Introduction}

In every cell, protein synthesis (namely translation) is mediated by the ribosome, a large macromolecular complex that decodes mRNAs into their corresponding sequences of aminoacids. Each particle is divided into two subunits: a large one that performs the links between the amino acids to form a polypeptide, and a small one that ensures the decoding of the messenger RNA (mRNA). During translation, ribosomes cluster into polysomes (or polyribosomes), in which they are bound together by mRNA. In this way many ribosomes simultaneously synthesize the same protein. It has been known for a while that polysomes adopt various spatial arrangements in vitro or in vivo. Among these, the most classical forms described are linear, spiral and helical ${ }^{1 ; 2 ; 3}$. In addition, two adjacent strands can assemble "back-to-back" or "front-to-back" to form polysomal hairpins or large spirals, respectively ${ }^{4}$. Interestingly, these spatial orientations are not randomly arranged, probably to deter nascent chain interactions between the consecutive ribosomes ${ }^{3}$. Though, the spatial organization of polysomes in the bacterial cell is far more complicated than previously expected from the absence of distinct functional compartments ${ }^{5}$. Indeed, the spatial distribution of the DNA and ribosomes is influenced by many parameters, including the crowding forces that condense the DNA into the nucleoid; the presence of nucleoid-associated proteins that bind and modify the DNA segments; the translational activity of the cell that leads to a modification in the amounts of ribosomes; and the mobility of translating ribosomes. How the translational machinery is organized under high spatial constraints is still poorly understood. The aim of our study was to better understand the way ribosomes organize when bacteria are submitted to a stress that accumulates multiple polysomes.

We first decided to work with Escherichia coli cells over-expressing an mRNA carrying a cluster of proline codons that are known to induce pausing during translation ${ }^{6 ;}$. We 
presumed that these conditions would saturate the rescue systems and lead to a marked accumulation of polysomes. However, despite a slowing-down of growth, the cells survived without a real modification in their polysomal profile. Therefore, we then used the same conditions but worked with a strain lacking tmRNA, which is the main actor of the "transtranslation" quality control system and allows for the release of stalled ribosomes. The cells again survived the hijacking of the translation machinery by the overexpressed problematic mRNAs. However, it resulted in a sharp modification of the organization of the ribosomal network. We analyzed this newly-modified organization using electron tomography, template matching, and 3D modeling. Our results demonstrate that single ribosomes are replaced with large amounts of compacted polysomes. These polysomes are highly organized in most cases, principally forming hairpins and dimers of hairpins that stack together in very compacted clusters, helping to maintain translation efficiency.

\section{Results}

\section{Overexpression of a protein carrying a stalling motif}

In this study, we first investigated cell growth of $E$. coli when overexpressing mRNAs carrying a run of proline codons. Indeed, the ribosome cannot synthesize all of the protein sequences equally well, and a stretch of prolines is likely to strongly inhibit elongation due to the perturbed geometry of peptidyl-transfer on proline residues ${ }^{7}$. We used the plasmid pET42a(+) that carries the gene encoding Glutathione S-Transferase (GST) followed by a cluster of 107 amino-acids with 7 CCA proline codons and 1 rare $(0.32 \%$ frequency) CUA leucine codon at its C-terminal end. For clarity, we refer to the protein of $\mathbf{7}$ proline codons as "GST7p" (Figure 1A). Our theory was that the stretch of seven prolines followed by one rare leucine codon would strongly impair translation and favor the accumulation of ribosomes into polysomes. Indeed, stalling of full-length proteins encoded by genes with efficient stop 
signals has been extensively observed and is particularly increased when the C-terminal sequence ends with Asp-Pro, Pro-Pro, or rare codons ${ }^{6}$.

We first examined the effect of GST7p induction on the growth of the E. coli wild-type strain MG1655. Cells were grown in LB medium until OD600 reached 0.4-0.6 and then the culture was split into two equal volumes. The expression of GST7p was induced in half the volume by the addition of IPTG (Figure 1B, black arrow) while the other half served as a control. Not surprisingly ${ }^{8}$, the cell growth dropped dramatically after the induction of protein synthesis (Figure 1B, compare dashed and solid lines), but the cell viability of the strain was only moderately affected (Figure 1C). Polysome profiling was then performed by centrifuging the cell extracts on sucrose gradients after $90 \mathrm{~min}$ of IPTG induction. The ribosomal profile is very similar before and after induction of the GST7p protein, as mainly 70S monoribosomes and almost no polysomes are recovered (Figure 1D). This suggests that such a situation does not counter-balance the rescuing systems, which therefore avoid the accumulation of polysomes ${ }^{9}$. Next we used the same conditions but worked with a strain lacking tmRNA, the main actor of the "trans-translation" quality control system that enables the release of stalled ribosomes $10 ; 11$.

\section{Overexpression of a protein carrying a stalling motif in the absence of the $\operatorname{tmRNA}$ rescuing factor}

Since a large network of factors dedicated to ribosome rescue ensure cell survival during stresses that induce ribosome stalling ${ }^{9}$, we decided to perform the same experiments in $\Delta s s r A$ cells lacking their first line of defense, the tmRNA-SmpB mediated reaction of transtranslation ${ }^{10 ; 11}$. Despite the absence of trans-translation, the growth curves and cell viability are identical to the ones recovered with the E. coli wild-type strain (compare Figures 2A and $2 \mathrm{~B}$ to $1 \mathrm{~B}$ and $1 \mathrm{C}$, respectively). Therefore, the $\Delta s s r A$ cells still survived the hijacking of the 
translation machinery by the problematic overexpressed mRNAs. Since ArfA (Alternative ribosome rescue factor A, formerly YhdL) is undoubtedly the primary tmRNA backup of tmRNA ${ }^{12}$, we figured that overexpression of the GST7p may be associated with an increase in its expression, thus ensuring ribosome rescue and cell growth in the absence of tmRNA. ArfA was detected by western blotting, and the variations in protein amounts between noninduced and induced cells were compared (Figure 2C). In comparison with wild-type E. coli cells harboring tmRNA, even before induction of bacterial stress due to overexpression of GST7p, the ssrA deletion led to an accumulation of high levels of ArfA ( 4-fold more than in wt cells) (Figure $2 \mathrm{C}$, line 0 ). This is in agreement with the known regulation of arfA mRNA expression by tmRNA tagging activity. ArfA is synthesized from truncated mRNA; therefore, in the absence of tmRNA, high amounts of the protein tend to accumulate ${ }^{13 ; 14}$. The comparison of ArfA levels between non-induced and induced cells revealed only an almost insignificant increase in the protein levels in the presence of GST7p $(\sim 1.3$-fold after $1 \mathrm{~h}$ induction and $\sim 1.1$-fold after $2 \mathrm{~h}$ induction). These data suggest that cell survival might be due to modifications other than a simple recruitment of the rescuing factor ArfA. ArfB (Alternative ribosome rescue factor B, formerly YaeJ) is not a good candidate, since it is not sufficient for recovering cell viability in the absence of tmRNA and ArfA ${ }^{15}$. Another possible rescuing mechanism could be supported by the elongation factor EF-P, recently shown to facilitate the translation of proline stretches ${ }^{16 ; 17}$. In that case, even in the absence of tmRNA, only a small number of polysomes should be recovered after over-expression of GST7p. We were thus particularly interested in examining the organization of the ribosomal fraction after cell induction, reasoning that an accumulation of polysomes on the problematic mRNAs would indicate that Arf systems or EF-P are not consistently recruited and that such an organization might participate in cell survival. 
Polysome profiling in $\triangle s s r A E$. coli cells after GST7p overexpression was then performed. As shown in Figure 2D, in the absence of stress mainly $70 \mathrm{~S}$ monoribosomes were recovered. Therefore, despite the absence of tmRNA, the alternative rescuing factors unquestionably take over the problematic mRNAs that accumulate in normal conditions. On the other hand, the translation of GST7p mRNA results in characteristic profiles including short and long polysomes (designated with 's' and ' 1 ', respectively), while $70 \mathrm{~S}$ monoribosomes can no longer be detected (Figure 2D). It must be noted that endogenous SmpB protein, tmRNA's usual partner, was mainly recovered linked to the ribosomal fractions in the presence or absence of tmRNA (data not shown and ${ }^{18}$ ), but it is not sufficient to cause the polysomal accumulation by itself as $70 \mathrm{~S}$ monoribosomes are predominant in non-induced $\Delta \mathrm{SsrA}$ cells (figure 2D, left). these results confirm that polysomes accumulate upon translation of the GST7p protein, and that neither alternative rescue factors (e.g., ArfA or ArfB) nor translation facilitators (e.g., EF-P) efficiently counterbalance their accumulation.

\section{Electron microscopy analysis of the polysomes}

We followed by using transmission electron microscopy (TEM) after negative staining to analyze the two main ' $s$ ' and ' $l$ ' polysomal fractions recovered on the sucrose gradients (Figure 2D). Both fractions contained mostly polysomes, with very scarce and isolated monoribosomes (Figure 3). The first zone contained the shortest polysomes, with several arranged in compacted clusters (Figure 3A, insets). Such short forms have already been described as being the initial step in polysome formation ${ }^{19}$. As expected, the longest polysomes were recovered on a higher density sucrose gradient fraction. Among these polysomes, most of the arrangements observed were classical linear strings with an average number of 4 to 6 ribosomes (Figure 3B, insets). Based on the size of the open reading frame of the mRNA encoding the GST7p protein (918 nts in the absence of any cleavage), and 
considering that the distance between two codon recognition sites in densely-packed neighboring ribosomes is estimated at $72 \mathrm{nts}{ }^{20}$, this means that up to 12 ribosomes can accumulate on a fully-extended GST7p mRNA. Therefore, although the polysomes observed after purification were shorter than the maximally-expected size, they imply an ongoing translation of the overproduced mRNA. To determine the presence of the mRNA carrying the run of proline codons in the polysomal fraction, we then performed RT-PCR analysis. We found that only the long polysomes harbored the mRNA (Figure 3C). These data are consistent with the distribution of non-stop mRNAs in a $\Delta s s r A$ E. coli strain $^{21}$. Other than linear strings, no other characteristic arrangements accumulated in these isolated polysomal fractions. This can be explained by a partial collapse of supramolecular arrangements during the TEM preparation so we next explored the polysomal organization in situ, using conditions as close as possible to the native state.

\section{Ultrastructure of $E$. coli cells when overexpressing the stalling motif}

To further explore the overall changes in cellular organization in conditions as close as possible to their native state, cells were subjected to high-pressure freezing and then freezesubstitution. This approach retains macromolecular structures with low artifacts, and enables ultrastructural observation of cytoplasmic domains such as polysomes while guarding their integrity ${ }^{22}$. The bacteria were frozen after 90 min of induction and compared with noninduced cells (Figure 4). The overexpression of GST7p obviously impacts the cellular organization, leading to an increase in the number of ribosomes (see also Figure 2D). The ribosomal over-crowding in the bacterial cytoplasm is associated with a much more restricted space occupied by the nucleoid (Figures 4A, B). The distribution of the GST7p protein within the cells was analyzed by immunogold labeling, with the protein marked with anti-GST (Figure 4C). The gold labeling appears uniformly-distributed throughout the cell, but in some 
places gold particles form small chains of 3-5 units that represent overlapping groups of consecutive ribosomes which could be interpreted as ongoing polysomal formation (Figure 4C, arrows). Accordingly, a strong production of GST7p proteins was detected by western blotting after induction by IPTG (Figure 4D). This confirms that the compaction of polysomes does not impair protein synthesis.

\section{Electron tomography and 3D organization of the polysomes}

For tomography analysis, tilt-series from -60 to +60 with an angular increment of $1^{\circ}$ were recorded. Figure $5 \mathrm{~A}$ presents the $0^{\circ}$ tilt image of the series. Densities corresponding to the 70S ribosome can already be observed forming compacted clusters. To characterize the 3D organization of the ribosomes, we then reconstructed the tomograms (Figure 5B). Considering that no independent ribosomes and only polysomes were recovered within the activated cells (see Figure 2C), we measured the spacing between the particles to distinguish one polysome from another. However, due to their very high level of compaction, the mean distances recovered between adjacent ribosomes were rather uniform and very often below $25 \mathrm{~nm}$. Therefore, this value cannot be used as an index to identify polysomes. Several characteristic supramolecular topologies are more frequently observed among this overcrowded population of polysomes that form very well-organized structures ${ }^{4 ;} 19$. Examples of these arrangements are shown in Figures 5A and 5B. Parallel double rows of ribosomes and tetramers are abundant, as clearly seen from our electron tomograms (Figure 5 and 6). To our knowledge, this is the first time that such compacted arrangements have been observed in living cells. From these tomograms, we used a template-matching approach with a $70 \mathrm{~S}$ ribosome to generate 3D models of the ribosomal arrangements (Figure 6, B, D, and E). Double rows of polysomes correspond to folded long polysomes forming hairpin-like structures (Figure 6B). These structures tend to pack together, yielding an overall topology of ribosomal tetramers 
(Figure 5B and 6C). These tetramers correspond to orthogonal views of two compacted polysome hairpins (Figure 6D and E).

\section{Discussion}

Electron tomography and template matching are state of the art tools for describing the threedimensional organization of large macromolecular complexes such as polysomes ${ }^{20 ; 23}$. Here for the first time we describe the formation of a very compacted population of polysomes. The formation of double-row polysomes has been described in eukaryotes as a long process requiring several rounds of translation. Double row polysomes also favor the reinitiation of translation, as do circular polysomes ${ }^{19}$. We assume that such a high concentration of polysome-rich regions will not favor the pairing of translation with transcription ${ }^{24}$, but that instead completed messages will accumulate in these regions for optimal protein synthesis through slow translation. This polysomal compaction would promote multiple rounds of translation of the same mRNA and help to avoid mRNA degradation by ribonucleases. This argues in favor of a non-random cellular reorganization of translation when actively-growing bacterial cells have to accommodate thousands of polysomes. The structures we observed are in agreement with the polysomal arrangements previously described by Baumeister's group ${ }^{20}$. Although we could not determine the exact configurations of neighboring orientations, it is interesting to note that even in this overcrowded environment the space available between the two rows of a tetramer is large enough $(>25 \mathrm{~nm})$ to minimize contacts between nascent chains in close proximity. This lack of contact may allow molecular chaperones to access the nascent chains.

Such an organization was obtained in the absence of trans-translation, the primary rescue system taking charge of stalled ribosomes.Several quality control mechanisms continuously ensure the accuracy of the process, particularly when the ribosomes are paused during mRNA 
translation. Much has been unraveled during recent years about the structure and functions of these bacterial rescuing systems, and we now have a better understanding of the processes that take place at the molecular level $15 ; 25 ; 26 ; 27$. However, at the ultrastructural level, little is known about how bacteria cope with protein synthesis when these rescuing systems are overwhelmed by the accumulation of problematic mRNAs. Our current findings strongly support the idea that when rescue factors (e.g, tmRNA-SmpB, ArfA, or ArfB) and translation facilitators (e.g., EF-P or EF4) ${ }^{28}$ cannot counterbalance the accumulation of polysomes, bacterial survival depends on the formation of a supramolecular network of polysomes in dedicated regions. The cellular organization in bacteria is more controlled and less random than previously thought ${ }^{29}$, and can successfully cope with a sudden accumulation of polysomes when facing translational stress that might otherwise rapidly overwhelm its quality control systems.

\section{Materials and Methods}

\section{Strains and plasmids}

Escherichia coli wild-type strain MG1655 or W3110 $\Delta s s r A$ (which lacks the tmRNA gene), ${ }^{30}$ were used in this study. The pET42a plasmid was obtained from Novagen (Merck Millipore, Nottingham, UK). It was co-transformed with the pACYC184 plasmid encoding the T7 RNA polymerase gene under the lac-promoter sequence into the $E$. coli strains. $\Delta s s r A E$. coli cells harboring pET42a plasmid driven by the $T 7$ promoter were used for the high-level production of non-stop mRNAs. Bacteria were grown in $\mathrm{LB}$ medium at $37^{\circ} \mathrm{C}$ in the presence of kanamycin and chloramphenicol antibiotics until $O D 600$ reached 0.4-0.6 and the expression of GST7p was induced by the addition of $1.0 \mathrm{mM}$ IPTG for $90 \mathrm{~min}$. The bacterial viability of the cultures was assayed by spotting LB agar plates with $5 \mu$ of ten-fold dilutions of cultures adjusted at $O D 600=0.5$. The plates were incubated at $37^{\circ} \mathrm{C}$ for $24 \mathrm{~h}$.

\section{Polysome extraction, purification and analysis}


Cell cultures were centrifuged at $4^{\circ} \mathrm{C}$ and the pellets resuspended in $5 \mathrm{~mL}$ of a polysome profile buffer (10mM Tris- $\mathrm{HCl} \mathrm{pH} 7.5,50 \mathrm{mM} \mathrm{NH}_{4} \mathrm{Cl}, 10 \mathrm{mM} \mathrm{MgCl}_{2}, 1 \mathrm{mM}$ DTT, $100 \mu \mathrm{g} / \mathrm{ml}$ chloramphenicol) then centrifuged a second time. After discarding the supernatant, the pellets were flash-frozen in liquid nitrogen. Lysis buffer (a polysome profile buffer containing $20 \mathrm{mg}$ lysozyme in a total volume of $2 \mathrm{ml}$ ) was added in the presence of $1 \mathrm{ml}$ of a detergent solution (DOC 10\%, Brij58 10\%, lysis buffer 1X, DNaseI). This mix was left on ice for 20 min before being centrifuged at $13,200 \mathrm{rpm}$ for $5 \mathrm{~min}$ at $4^{\circ} \mathrm{C}$. The supernatants were then filtered on a $0.45 \mu \mathrm{m}$ membrane. $10-40 \%$ sucrose gradients were prepared under standard conditions and used for polysomal fractionation. Ultracentrifugation was performed at 41,600 rpm for $3 \mathrm{~h}$ at $4^{\circ} \mathrm{C}$ in a SW55 rotor (Beckman-Coulter, Villepinte, France). Then the gradients were separated into 20 fractions and analyzed. To perform electron microscopy imaging, the polysomes were further purified by ultracentrifugation in $20 \%$ sucrose cushions rotating at $29,000 \mathrm{rpm}$ for $3 \mathrm{~h}$ at $4^{\circ} \mathrm{C}$ in a SW55 rotor (Beckman-Coulter, Villepinte, France). The supernatants were then carefully discarded and the pellets resuspended in $100 \mu \mathrm{l}$ of polysome profile buffer. Aliquots of $10 \mu \mathrm{l}$ were conserved at $-80^{\circ} \mathrm{C}$ before being observed. RT-PCR analysis was performed on $2 \mu 1$ of short or long polysomal fractions using SuperScript ${ }^{\mathrm{TM}}$ II Reverse Transcriptase (Invitrogen) and GoTaq ${ }^{\circledR}$ (Promega), according to the manufacturers’ protocols. Primers used for RT-PCR were as follows: GST RT, 5'-TCCATG TGCTGGCGTTCG-3'; GST forward, 5'-ATGTCCCCTATACTAGGT-3'; and GST reverse, 5'-TGGTGGTGCTCGAGTGCG-3'. Note that aspecific bands could be detected on the short polysomal fraction only. Since this ribosomal fraction is highly heterogeneous and does not contain the GSTp mRNA, we presume that these bands are 16S and 23S ribosomal RNAs.

\section{High pressure freezing / freeze substitution}

Cell slurry was prepared by re-suspending E. coli cells in 20\% Bovine Serum Albumin (BSA) in LB medium. A $2 \mu \mathrm{l}$ drop of the pellet was loaded onto a pre-heated $0.5 \mathrm{~mm}$-thick flat specimen carrier having a gold-plated surface and a cavity diameter of $1.5 \mathrm{~mm}$ and depth of $0.2 \mathrm{~mm}$ (Cat. No 16706898, Leica Microsystems AG, Wetzlar, Germany). These were then frozen in a Leica EM PACT2 high-pressure freezer. Frozen samples in the carriers were immediately transferred to an automatic freeze substitution system (Leica EM AFS2) equipped with an automatic reagent handling system (Leica EM FSP), and were placed in the pre-cooled $-90^{\circ} \mathrm{C}$ substitution solution. Cells were freeze-substituted in acetone containing 
$0.1 \%$ uranyl acetate at $-90^{\circ} \mathrm{C}$ for $60 \mathrm{~h}$. After that, the temperature was elevated at a rate of $3^{\circ} \mathrm{C} / \mathrm{h}$ to $-50^{\circ} \mathrm{C}$, and samples were kept at this temperature for $24 \mathrm{~h}$. Then the samples were washed once with acetone and three times with $100 \%$ ethanol at $-50^{\circ} \mathrm{C}$. Samples were infiltrated with resin/100\% ethanol mixtures by raising the volume-to-volume proportion of Lowicryl HM20 (25\% for 2 h, 50\% for 2 h, $75 \%$ overnight, and $100 \%$ four times for $1 \mathrm{~h}$ ). Finally, polymerization was carried out at $-50^{\circ} \mathrm{C}$ for $48 \mathrm{~h}$ and at $20^{\circ} \mathrm{C}$ for $48 \mathrm{~h}$. Ultra-thin (90$110 \mathrm{~nm}$ ) sections were collected on nickel grids (300 mesh).

\section{Electron Tomography}

Tilt-series were recorded on a Tecnai G2 Sphera transmission electron microscope (FEI Company, Eindhoven, Netherlands) operating at $200 \mathrm{kV}$ with a nominal magnification of x25000. Digital images were collected with a 2k x 2k Megascan CCD camera. Tilt-series of 80- to 100-nm-thick sections were recorded from -60 to +60 with an angular increment of $1^{\circ}$. The eTomo graphical user interface of the IMOD Tomography package ${ }^{31}$ was used to calculate the $3 \mathrm{D}$ reconstruction. Images were pre-processed, binned, and aligned. CTF correction and 2D filtering were applied on the tilt-series before reconstruction. The 3D reconstructions were performed by weighted back-projection. The tomogram resolution, estimated at $38 \AA$, was calculated using the McEwen and Marko formula specially designed for resin-embedded samples ${ }^{32}$.

\section{Template Matching}

To identify the ribosomes in tomograms, we employed a template-matching approach based on the measure of the correlation between the template (density map of a reference particle) and the tomogram ${ }^{33 ; 34}$. Using the IMAGIC software, a template was generated from an atomic model of the E. coli 70S ribosome (PDB ID: 3IZU and 3IZW) by down-sampling to the same pixel size as the tomogram and filtering to a similar resolution. All files were converted into EM format using TOM Toolbox ${ }^{35}$ in MATLAB. The template-matching process was completed using MOLMATCH software ${ }^{33}$, with an angular increment of $10^{\circ}$. Cross-correlation maxima were extracted with the Av3 Toolbox ${ }^{36}$ in MATLAB while using a minimum peak radius slightly larger than that of the $70 \mathrm{~S}$ ribosome. This resulted in a set of potential particles that were defined by their coordinates $(\mathrm{x}, \mathrm{y}, \mathrm{z})$ and relative orientations $(\varphi, \psi, \theta)$. Cross-correlation values ranged between 0.49 and 0.65 . 


\section{Immuno-electron microscopy}

Lowicryl HM20-embedded thin sections of E. coli cells were first blocked with $1 \%$ BSA in $100 \mathrm{mM}$ (pH 7.4) Phosphate buffer saline (PBS) for $2 \mathrm{~h}$ and $30 \mathrm{~min}$ and then reacted for $2 \mathrm{~h}$ with primary antibody diluted in blocking buffer. Rabbit anti-GST antibodies were diluted at 1:250. Following four washes with blocking buffer (10 min each time) and one wash in $100 \mathrm{mM}$ Tris- $\mathrm{HCl}$ ( $\mathrm{pH} 7.4$ ) containing 1\% BSA, we incubated the grids for $1 \mathrm{~h}$ with the second antibody coupled with $10 \mathrm{~nm}$ gold particles (dilution 1:40, Delta Microscopies, Ayguesvives, France). After subsequent washes with Tris- $\mathrm{HCl}$ ( $\mathrm{pH}$ 7.4) buffer containing 1\% BSA, grids were fixed with $2.5 \%$ glutaraldehyde and contrasted for $40 \mathrm{~min}$ in $5 \%$ uranyl acetate.

\section{Negative staining}

Following polysome separation, samples were diluted 10 times in polysome profile buffer and applied to freshly glow-discharged 300-mesh collodion/carbon coated grids. After three washes in this buffer, grids were stained with $2 \%$ uranyl acetate for 30 seconds. The grids were then observed with a Tecnai G2 Sphera transmission electron microscope operating at $200 \mathrm{kV}$. Images were recorded with a $4 \mathrm{k}$ x $4 \mathrm{k}$ Ultrascan CCD camera at a nominal magnification of $\mathrm{x} 29000$.

\section{Western blotting}

Following IPTG induction, $1 \mathrm{ml}$ of cells were pelleted and resuspended in $50 \mu \mathrm{l}$ of lysis buffer (Tris-EDTA pH=8, 1mM PMSF, 0.15\% Sodium Deoxycholate, 0.01\% SDS) for 15 min at $37^{\circ} \mathrm{C}$. After centrifugation, SDS was added to the supernatant for a final concentration of $1 \%$, and sample concentrations were measured by Bradford Assay. $10 \mu \mathrm{g}$ of protein extract was loaded on a 15\% SDS-PAGE and transferred onto a PVDF membrane (Biorad Laboratories, USA). Western blot analysis was performed using anti-ArfA antibodies (provided by Pr T. Abo) at 1:1000 or anti-GST antibodies (Bethyl Laboratory, Montgomery, USA) at $1: 1000$.

\section{Acknowledgements}

This work was supported by the French National Agency [ANR-08JCJC-0027-01 and ANR09-MIE to R.G.] and the Ligue Nationale Contre le Cancer [to N.C.]. We are grateful to Dr. F. Förster (Martinstried, Germany) for supplying us with MOLMATCH Software and the TOM 
Toolbox, to Drs. Tatsuhiko Abo (Okayama, Japan), Marc Hallier and Brice Felden (Rennes, France) for providing us with the anti-ArfA and anti-SmpB antibodies, respectively. EM experiments were processed at the Microscopy Rennes Imaging Center (MRic) facilities (http://microscopie.univ-rennes1.fr/). 


\section{Figures and Legends}

A

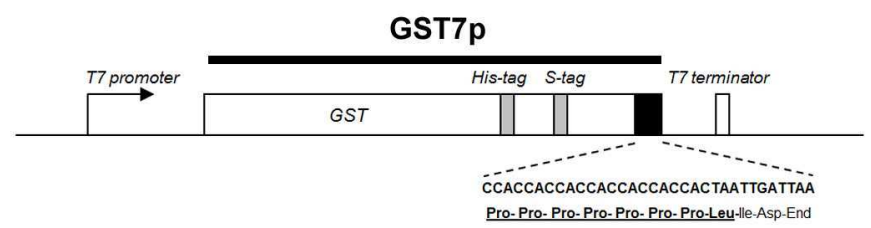

B

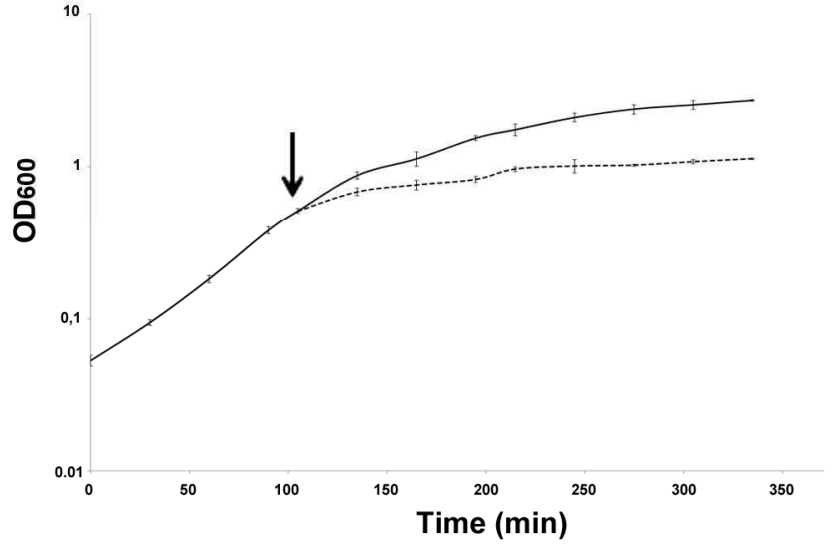

C

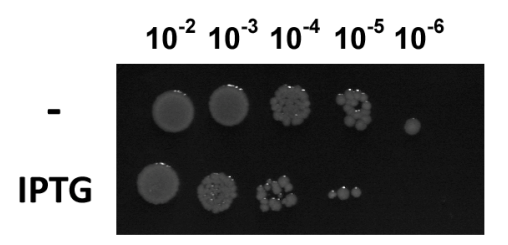

D

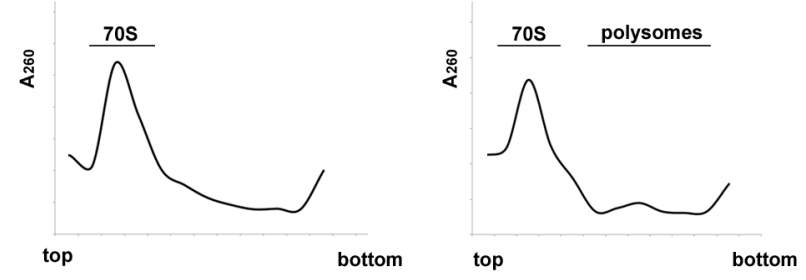

Figure 1. Growth curve analysis of $E$. coli wild-type strain expressing GST7p.

A) Schematic diagram of the construct used. The cluster of proline codons is underlined. B) Growth of E. coli strains was conducted at $37^{\circ} \mathrm{C}$. Samples are: Solid line: non-induced MG1655/pET42a E. coli; dashed line: same strain, induced by IPTG at $O D 600=0.5$ (black arrow). Triplicate cultures were conducted for each experiment. C) Spots (5 $\mu$ l) of 10-fold dilutions of induced cultures (IPTG) versus non-induced cultures (-) incubated at $37^{\circ} \mathrm{C}$ for 24 h. D) Sucrose gradient sedimentation profiles of ribosomes upon overexpression of GST7p. Ribosome analysis was performed by centrifugation of the cell extracts on linear $10-40 \%$ sucrose density gradients (see experimental procedures), and the absorption profiles measured at $260 \mathrm{~nm}$. The zones containing monosomes (70S) and polysomes are indicated. Left: Ribosome profiles in non-induced cells. Right: Ribosome profiles in cells after 90 min IPTG induction. 
A

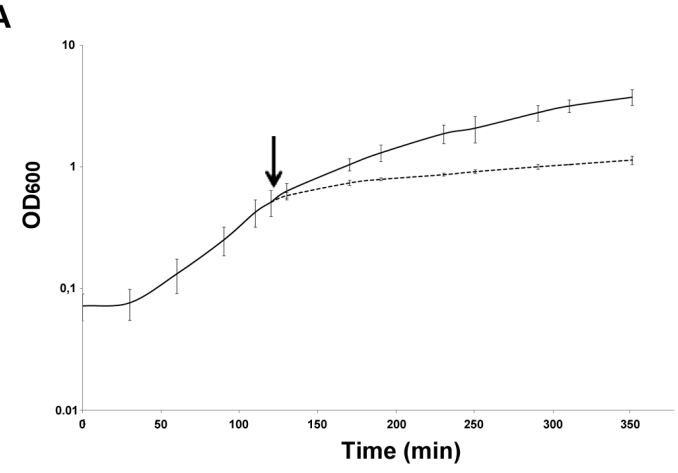

B

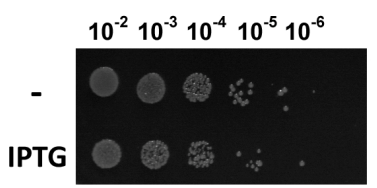

C

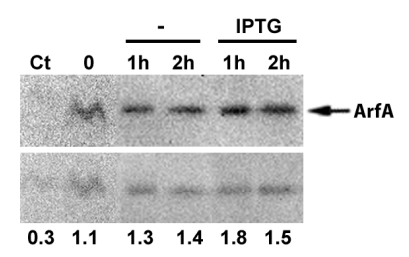

D

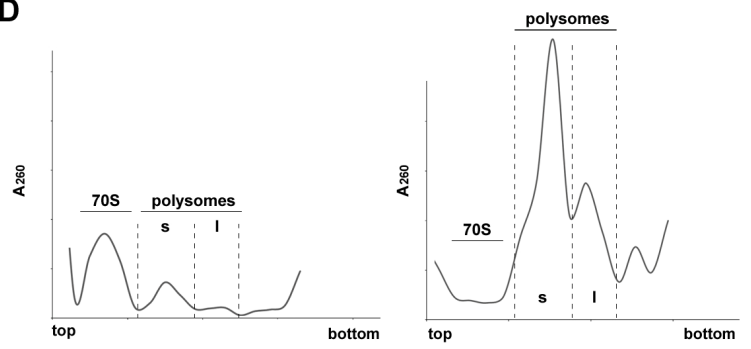

Figure 2. Growth curve analysis of $E$. coli $\Delta s s r A$ strain expressing GST7p.

A) Growth of E. coli strains was conducted at $37^{\circ} \mathrm{C}$. Samples are: Solid line: non-induced W3110 $\Delta s s r A$ /pET42a E. coli; dashed line: same strain, induced by IPTG at $O D 600=0.5$ (black arrow). Triplicate cultures were conducted for each experiment. B) Spots (5 $\mu 1)$ of 10fold dilutions of induced cultures (IPTG) versus non-induced cultures (-) incubated at $37^{\circ} \mathrm{C}$ for 24h. C) Representative membrane of several independent western blots of the total E. coli extracts for ArfA protein. Cells were submitted to WB analysis either 1 or 2 hours after the time of induction in non-induced (-) or induced (IPTG) cells, respectively. Key: Ct, control corresponding to $E$. coli $\mathrm{DH} 5 \alpha ; \mathbf{0}, \Delta \mathrm{SSrA} E$. coli before induction. A non-specific band (lower panel) detected with the same antibodies was used as loading control. D) Sucrose gradient sedimentation profiles of ribosomes upon overexpression of GST7p. Ribosome analysis was performed by centrifugation of the cell extracts on linear 10-40\% sucrose density gradients (see experimental procedures), and the absorption profiles measured at $260 \mathrm{~nm}$. The zones containing monosomes (70S) and polysomes of short (s) and long (l) sizes are indicated. Left: ribosome profiles in non-induced cells. Right: ribosome profiles in cells after 90-minute IPTG induction. 

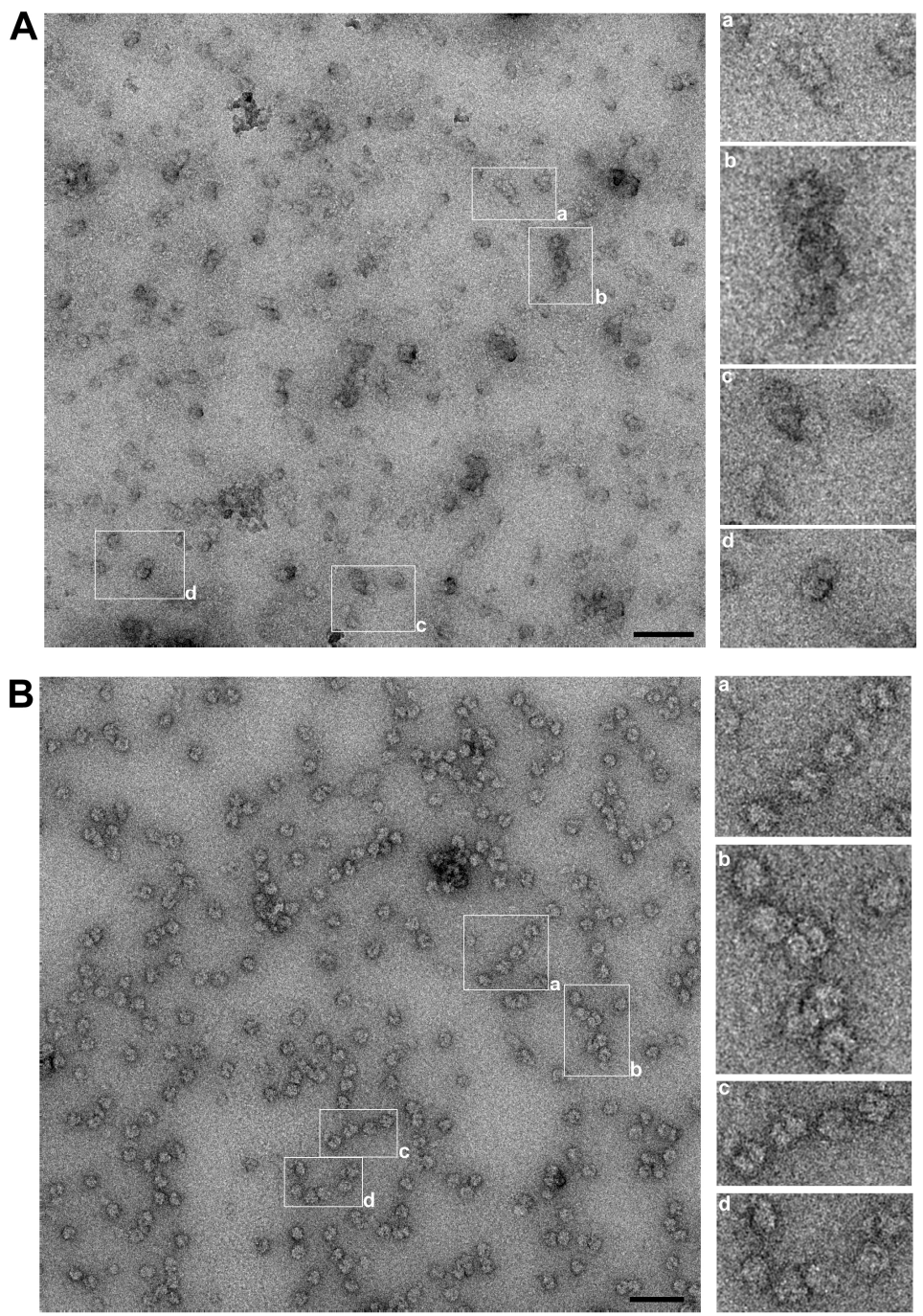

C
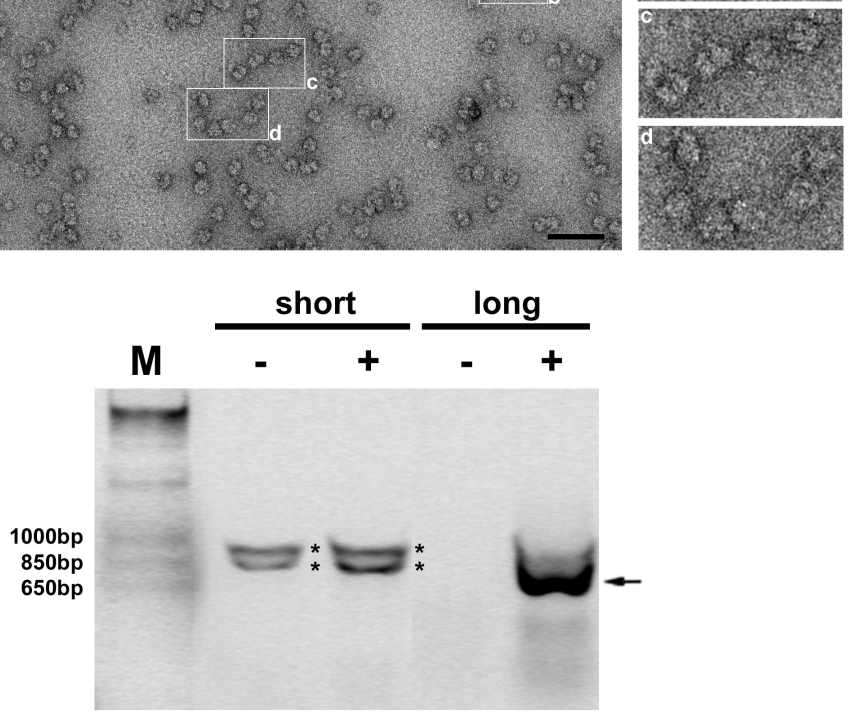

Figure 3. EM micrographs of monosomes and polysomes negatively stained with $2 \%$ uranyl acetate. A) Gradient fraction corresponding to short polysomes (s). B) Gradient fraction corresponding to long polysomes (1). Linear strings of 6-8 ribosomes and single ribosomes are observed. The presence of such monosomes in the fraction must result from a partial collapse of polysomes during TEM preparation. Scale bars, $100 \mathrm{~nm}$. C) Detection of the GST7p mRNA in the polysomal fractions by RT-PCR analysis. Key: M, DNA marker (base pairs); (-), negative control (PCR without reverse transcription); (+) RT-PCR product; arrow, band corresponding to GST7p mRNA; *: aspecific bands. 


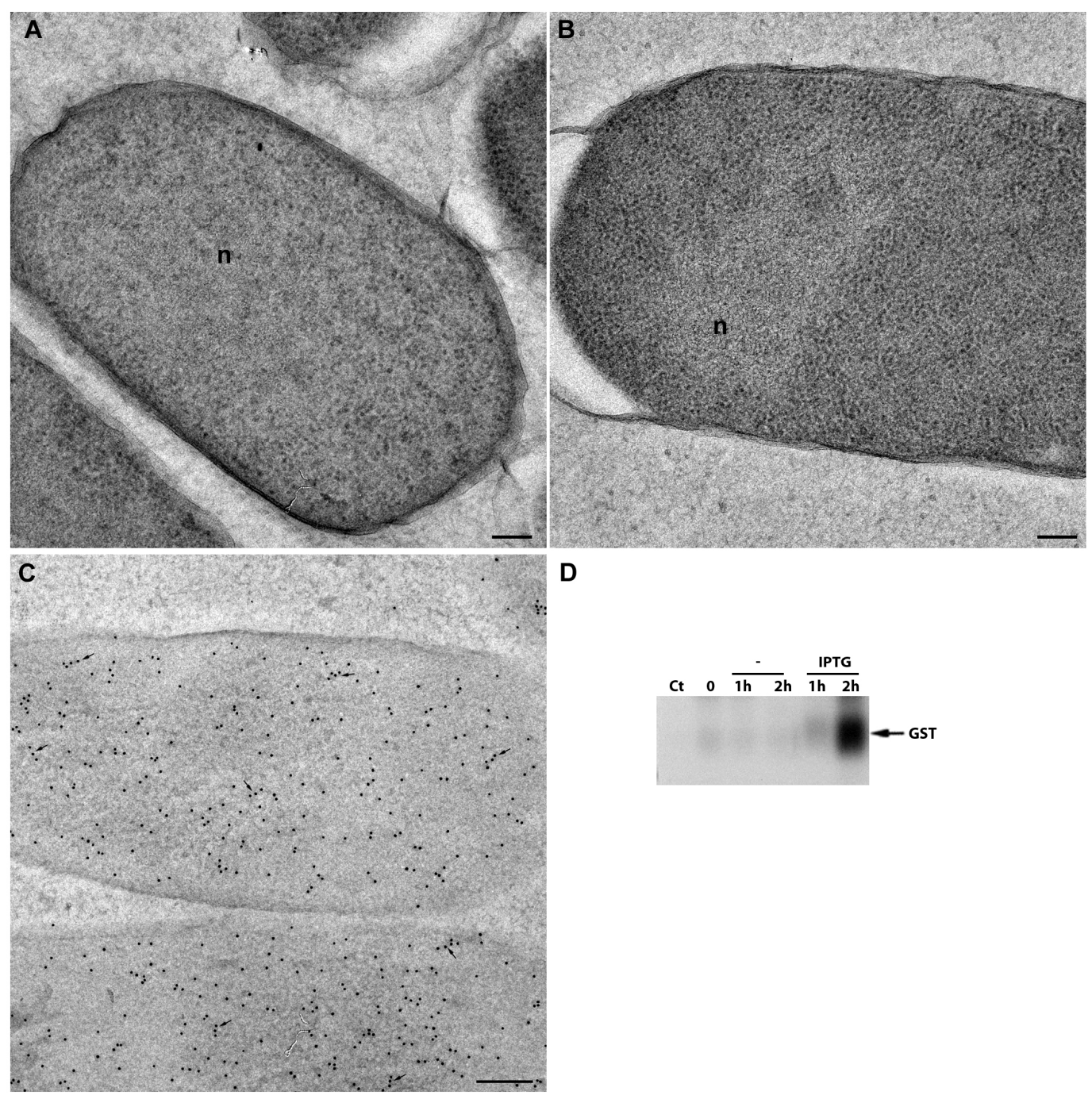

Figure 4. Thin sections of $\boldsymbol{E}$. coli $\Delta s s r A$ cells expressing GST7p. A) In control experiments, bacteria possess an evenly-distributed cytoplasm packed with ribosomes and a scattered nucleoid (n). B) After IPTG induction, bacteria have a compacted nucleoid (n) and a cytoplasm over-crowded with ribosomes, some of which form linear arrangements. C) Immunogold labeling with anti-GST on IPTG-induced cells. Most of the gold labeling is uniformly-distributed throughout the cell, however small gold chains overlap groups of consecutive ribosomes and should correspond to synthesizing polyribosomes (arrows). Scale bars, $100 \mathrm{~nm}$. D) Representative membrane of several independent western blots of the total E. coli extracts for GST protein. Cells were submitted to WB analysis either 1 or 2 hours after the time of induction in non-induced (-) or induced (IPTG) cells, respectively. Key: Ct, control corresponding to E. coli DH5 $\alpha ; \mathbf{0}, E$. coli $\triangle S S r A$ strain without induction 

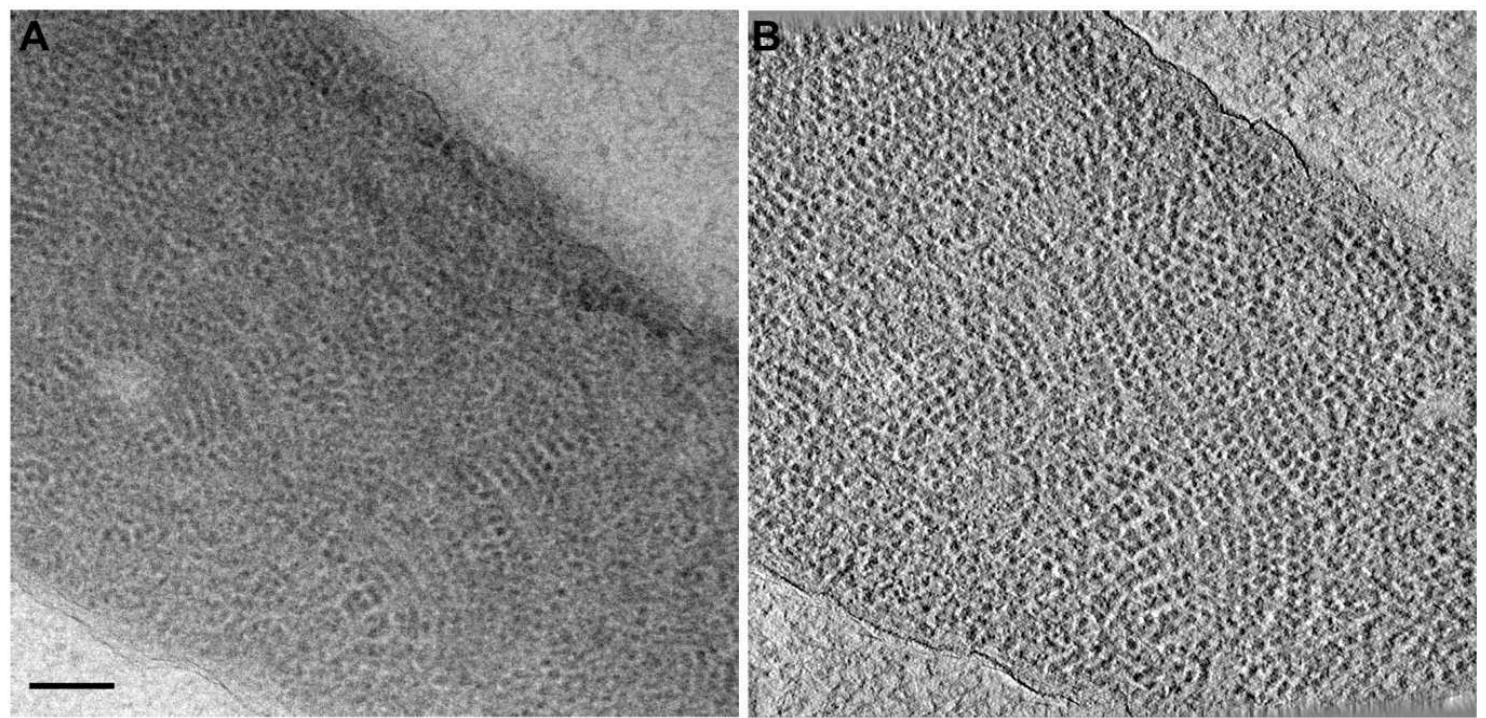

Figure 5. Electron tomography on $100 \mathrm{~nm}$-thick sections of $E$. coli $\Delta s s r A$ cells expressing GST7p. A) Image of the $0^{\circ}$ tilt series. Chains of compacted polysomes are clearly observed. B) Central section of the reconstructed tomogram. Polysomes form double row units or assemble as tetramers. Scale bar, $100 \mathrm{~nm}$. 


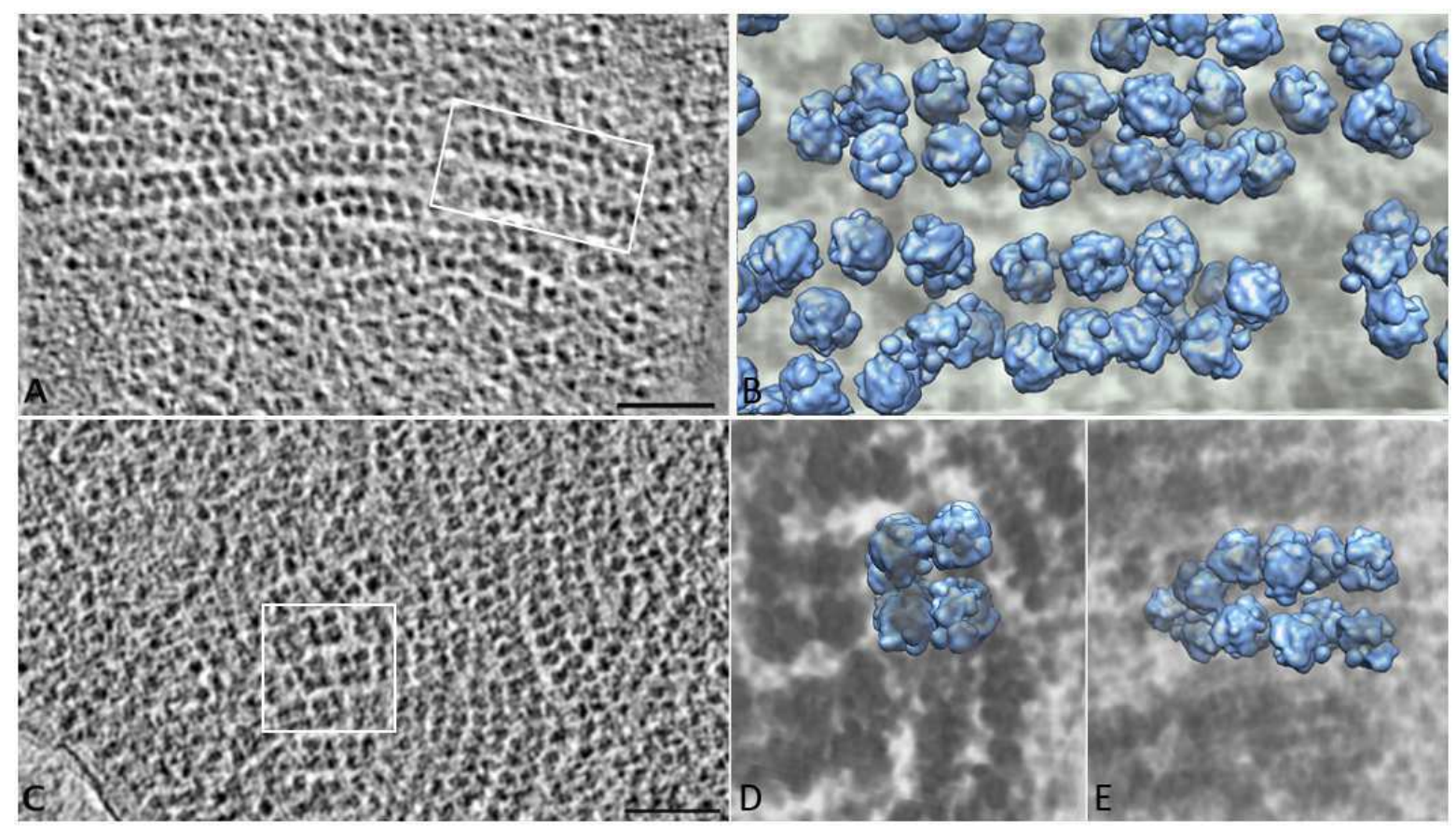

Figure 6. Electron tomography on $E$. coli $\Delta s s r A$ cells expressing GST7p and 70S template matching. A) and C) represent the central section of 2 tomograms. B), D) and E) are the whole volumes corresponding to the insets in $\mathbf{A}$ ) and $\mathbf{C}$ ). B) and D) represent views perpendicular to the tomographic $Z$ axis, while $\mathrm{E}$ ) is the same as D) but viewed from an axis parallel to the $Z$ axis. The $70 \mathrm{~S}$ ribosomal templates highlight the spatial arrangement of polysomes. One double-row polysome corresponds to a folded long polysome forming a hairpin-like structure (B, E). Tetramers (D) are orthogonal views of two compacted hairpins (E). Scale bars, $100 \mathrm{~nm}$. 


\section{References}

1. Blobel, G. \& Dobberstein, B. (1975). Transfer of proteins across membranes. II. Reconstitution of functional rough microsomes from heterologous components. $J$ Cell Biol 67, 852-62.

2. Slayter, H. S., Warner, J. R., Rich, A. \& Hall, C. E. (1963). The Visualization of Polyribosomal Structure. J Mol Biol 7, 652-7.

3. Brandt, F., Carlson, L. A., Hartl, F. U., Baumeister, W. \& Grunewald, K. (2010). The three-dimensional organization of polyribosomes in intact human cells. Mol Cell 39, 560-9.

4. Christensen, A. K. \& Bourne, C. M. (1999). Shape of large bound polysomes in cultured fibroblasts and thyroid epithelial cells. Anat Rec 255, 116-29.

5. Campos, M. \& Jacobs-Wagner, C. (2013). Cellular organization of the transfer of genetic information. Curr Opin Microbiol 16, 171-6.

6. Hayes, C. S., Bose, B. \& Sauer, R. T. (2002). Proline residues at the C terminus of nascent chains induce SsrA tagging during translation termination. J Biol Chem 277, 33825-32.

7. Woolstenhulme, C. J., Parajuli, S., Healey, D. W., Valverde, D. P., Petersen, E. N., Starosta, A. L., Guydosh, N. R., Johnson, W. E., Wilson, D. N. \& Buskirk, A. R. (2013). Nascent peptides that block protein synthesis in bacteria. Proc Natl Acad Sci U $S$ A 110, E878-87.

8. Mairhofer, J., Scharl, T., Marisch, K., Cserjan-Puschmann, M. \& Striedner, G. (2013). Comparative Transcription Profiling and In-Depth Characterization of Plasmid-Based and Plasmid-Free Escherichia coli Expression Systems under Production Conditions. Appl Environ Microbiol 79, 3802-12.

9. Giudice, E. \& Gillet, R. (2013). The task-force that rescues stalled ribosomes in eubacteria. Trends in Biochemical Sciences.

10. Moore, S. D. \& Sauer, R. T. (2007). The tmRNA system for translational surveillance and ribosome rescue. Annu Rev Biochem 76, 101-24.

11. Felden, B. \& Gillet, R. (2011). SmpB as the handyman of tmRNA during transtranslation. RNA Biol 8, 440-9.

12. Chadani, Y., Ono, K., Ozawa, S., Takahashi, Y., Takai, K., Nanamiya, H., Tozawa, Y., Kutsukake, K. \& Abo, T. (2010). Ribosome rescue by Escherichia coli ArfA (YhdL) in the absence of trans-translation system. Mol Microbiol 78, 796-808.

13. Chadani, Y., Matsumoto, E., Aso, H., Wada, T., Kutsukake, K., Sutou, S. \& Abo, T. (2011). trans-translation-mediated tight regulation of the expression of the alternative ribosome-rescue factor ArfA in Escherichia coli. Genes Genet Syst 86, 151-63.

14. Garza-Sanchez, F., Schaub, R. E., Janssen, B. D. \& Hayes, C. S. (2011). tmRNA regulates synthesis of the ArfA ribosome rescue factor. Mol Microbiol 80, 1204-19.

15. Chadani, Y., Ono, K., Kutsukake, K. \& Abo, T. (2011). Escherichia coli YaeJ protein mediates a novel ribosome-rescue pathway distinct from SsrA- and ArfA-mediated pathways. Mol Microbiol 80, 772-85.

16. Doerfel, L. K., Wohlgemuth, I., Kothe, C., Peske, F., Urlaub, H. \& Rodnina, M. V. (2013). EF-P is essential for rapid synthesis of proteins containing consecutive proline residues. Science 339, 85-8.

17. Ude, S., Lassak, J., Starosta, A. L., Kraxenberger, T., Wilson, D. N. \& Jung, K. (2013). Translation elongation factor EF-P alleviates ribosome stalling at polyproline stretches. Science 339, 82-5. 
18. Hallier, M., Ivanova, N., Rametti, A., Pavlov, M., Ehrenberg, M. \& Felden, B. (2004). Pre-binding of small protein B to a stalled ribosome triggers trans-translation. $J$ Biol Chem 279, 25978-85.

19. Kopeina, G. S., Afonina, Z. A., Gromova, K. V., Shirokov, V. A., Vasiliev, V. D. \& Spirin, A. S. (2008). Step-wise formation of eukaryotic double-row polyribosomes and circular translation of polysomal mRNA. Nucleic Acids Res 36, 2476-88.

20. Brandt, F., Etchells, S. A., Ortiz, J. O., Elcock, A. H., Hartl, F. U. \& Baumeister, W. (2009). The native 3D organization of bacterial polysomes. Cell 136, 261-71.

21. Kuroha, K., Horiguchi, N., Aiba, H. \& Inada, T. (2009). Analysis of nonstop mRNA translation in the absence of tmRNA in Escherichia coli. Genes Cells 14, 739-49.

22. Cougot, N., Cavalier, A., Thomas, D. \& Gillet, R. (2012). The dual organization of Pbodies revealed by immunoelectron microscopy and electron tomography. J Mol Biol 420, 17-28.

23. Myasnikov, A. G., Afonina, Z. A. \& Klaholz, B. P. (2013). Single particle and molecular assembly analysis of polyribosomes by single- and double-tilt cryo electron tomography. Ultramicroscopy 126, 33-9.

24. Bakshi, S., Siryaporn, A., Goulian, M. \& Weisshaar, J. C. (2012). Superresolution imaging of ribosomes and RNA polymerase in live Escherichia coli cells. Mol Microbiol 85, 21-38.

25. Gagnon, M. G., Seetharaman, S. V., Bulkley, D. \& Steitz, T. A. (2012). Structural basis for the rescue of stalled ribosomes: structure of YaeJ bound to the ribosome. Science 335, 1370-2.

26. Neubauer, C., Gillet, R., Kelley, A. C. \& Ramakrishnan, V. (2012). Decoding in the absence of a codon by tmRNA and SmpB in the ribosome. Science 335, 1366-9.

27. Weis, F., Bron, P., Giudice, E., Rolland, J. P., Thomas, D., Felden, B. \& Gillet, R. (2010). tmRNA-SmpB: a journey to the centre of the bacterial ribosome. EMBO J 29, 3810-8.

28. Giudice, E. \& Gillet, R. (2013). The task force that rescues stalled ribosomes in bacteria. Trends Biochem Sci 38, 403-11.

29. Trevors, J. T., Elsas, J. D. \& Bej, A. K. (2012). The Molecularly Crowded Cytoplasm of Bacterial Cells: Dividing Cells Contrasted with Viable but Non-culturable (VBNC) Bacterial Cells. Curr Issues Mol Biol 15, 1-6.

30. Komine, Y., Kitabatake, M., Yokogawa, T., Nishikawa, K. \& Inokuchi, H. (1994). A tRNA-like structure is present in 10Sa RNA, a small stable RNA from Escherichia coli. Proc Natl Acad Sci U S A 91, 9223-7.

31. Kremer, J. R., Mastronarde, D. N. \& McIntosh, J. R. (1996). Computer visualization of three-dimensional image data using IMOD. J Struct Biol 116, 71-6.

32. McEwen, B. F. \& Marko, M. (2001). The emergence of electron tomography as an important tool for investigating cellular ultrastructure. J Histochem Cytochem 49, 55364.

33. Forster, F., Han, B. G. \& Beck, M. (2010). Visual proteomics. Methods Enzymol 483, 215-43.

34. Frangakis, A. S., Bohm, J., Forster, F., Nickell, S., Nicastro, D., Typke, D., Hegerl, R. \& Baumeister, W. (2002). Identification of macromolecular complexes in cryoelectron tomograms of phantom cells. Proc Natl Acad Sci U S A 99, 14153-8.

35. Nickell, S., Forster, F., Linaroudis, A., Net, W. D., Beck, F., Hegerl, R., Baumeister, W. \& Plitzko, J. M. (2005). TOM software toolbox: acquisition and analysis for electron tomography. J Struct Biol 149, 227-34.

36. Forster, F. \& Hegerl, R. (2007). Structure determination in situ by averaging of tomograms. Methods Cell Biol 79, 741-67. 
Polysomal compaction in bacteria 